\title{
Influence of the initially ordered structure on transition of structural pattern
}

\author{
Chin-Yih Hong ${ }^{\mathrm{a}, *}$, P.C. Huang ${ }^{\mathrm{a}}$, Chun-Hui Chen ${ }^{\mathrm{b}}$, S.Y. Yang ${ }^{\mathrm{b}}$, \\ H.E. Horng ${ }^{\mathrm{b}}$, H.C. Yang \\ ${ }^{a}$ Department of Mechanical Engineering, Da-Yeh University, Chang-Hwa 515, Taiwan \\ ${ }^{\mathrm{b}}$ Department of Physics, National Taiwan Normal University, Taipei 116, Taiwan \\ ${ }^{\mathrm{c}}$ Department of Physics, National Taiwan University, Taipei 106, Taiwan
}

\begin{abstract}
The influence of the initially ordered state to the structural evolution on the magnetic fluid film under perpendicular magnetic fields was investigated. The magnetic field was increased from zero to a certain strength $H_{\mathrm{i}}$ at a given sweep rate $\mathrm{d} H / \mathrm{d} t$ and maintained at $H_{\mathrm{i}}$ for a period of time, after which the magnetic field was further raised at $\mathrm{d} H / \mathrm{d} t$. The $H_{\mathrm{i}}$ was adequately set so that a granular ordered structure of magnetic columns formed in the magnetic fluid film. This granular ordered structure served as an initial state for the following structural evolution when the magnetic field was increased from $H_{\mathrm{i}}$. When the magnetic field was raised up to $H_{\mathrm{s}}$, the structure evolved to the transition state, at which each column split into two columns. To obtain the initial structures of various orderness, the duration time $t_{\mathrm{d}}$ at $H_{\mathrm{i}}$ was varied from 1 to $180 \mathrm{~s}$. It was found that the degree of orderness of the initial structure under $H_{\mathrm{i}}$ increases with $t_{\mathrm{d}}$, and a higher $H_{\mathrm{s}}$ was also observed. This result can be interpreted by taking the interactions between a column and its neighbors into account.
\end{abstract}

(C) 2002 Elsevier Science B.V. All rights reserved.

Keywords: Magnetic fluid; Initial state; Duration time; Transition state

\section{Introduction}

Pattern forming systems of magnetic fluid films under perpendicular magnetic fields have recently attracted scientific interest. For this area of study, a variety of different pattern forming systems of magnetic fluid films have been studied, such as the geometry of agglomeration of magnetic fluids assuming a pattern of uniform cylinders of equal height and diameter [1], and quasitwo-dimensional periodic lattices formed under the influence of a perpendicular magnetic field [2]. Also, the formative process of ordered structure in magnetic fluid films has been reported, and in these the structural pattern evolves from a disordered column phase to a hexagonal structure, and then to a labyrinthine pattern [3]. Later, the transition from the disordered columns to

\footnotetext{
*Corresponding author.

E-mail address: cyhong@mail.dyu.edu.tw (C.-Y. Hong).
}

the ordered structure in the magnetic fluid film under the application of an external magnetic field was further proved with the observation of the diffraction of an incident parallel white light [4]. In contrast to that reported in Ref. [3], the hexagonal structural pattern reached the next-level hexagonal structural pattern through a phase transition instead of becoming a labyrinthine pattern [5]. Others also investigated the effect of lapsing time during the particle aggregation in magnetic fluid film [6,7]. Under a linearly applied perpendicular magnetic field, the macro-cluster areal density of magnetic fluid films depends upon the initial ordering of macro-clusters and upon the details of the magnetic field application sequence [6]. Hence, the effect of time during the earlier stage is crucial to the formative process of the ordered structures in magnetic fluid films. Also, in Ref. [7], a phase diagram, in the $H-H_{\mathrm{i}}$ plane, based on the effect of the initial state to the structural pattern in magnetic fluid films under perpendicular 
magnetic field was constructed [7], in which the initial states were generated by varying the initial field strength $H_{\mathrm{i}}$ with the same duration time, $t_{\mathrm{d}}=3 \mathrm{~min}$, for each $H_{\mathrm{i}}$. In this diagram, the phase transition of the ordered structural pattern, in which the magnetic columns split, also changed due to the variation of the initial state. Since the lapsing time during the formation process affects the particle aggregation, it is to be expected that the duration time $t_{\mathrm{d}}$ at the initial field strength $H_{\mathrm{i}}$ would have similar effect on the transition phase as well.

\section{Experimental details}

The homogeneous kerosene-based magnetic fluid $\left(\mathrm{MnFe}_{2} \mathrm{O}_{4}\right)$ with saturated magnetization of $8.12 \mathrm{emu} / \mathrm{g}$ used in this study was prepared with the co-precipitation technique. To form a magnetic fluid thin film, the magnetic fluid was dropped into a glass cell of thickness $6 \mu \mathrm{m}$ and then sealed with a glass cover plate. In this experiment, a pair of solenoids was used to generate uniform magnetic fields perpendicular to the planes of the films and the temperature was kept constant throughout each experiment by circulating cooling water around the solenoids. The photo images of the structural pattern were taken using an optical microscope and recorded in a personal computer. These photo images were converted to images in k-space by using fast Fourier transform (FFT) transformation. Generally, if the original structural pattern is a perfectly ordered hexagonal structure, the image in k-space will show six black spots forming a hexagonal pattern. In contrast, if the pattern is not so ordered, the intensity of the black spots disperses radially as well as circumferentially and sometimes forms a wide-band ring. Thus, the FFT images of the structural patterns could be used to compare the orderness of the initial states qualitatively.

\section{Results and discussion}

Fig. 1 gives a typical phase diagram of the structural pattern, formed inside a magnetic fluid film under

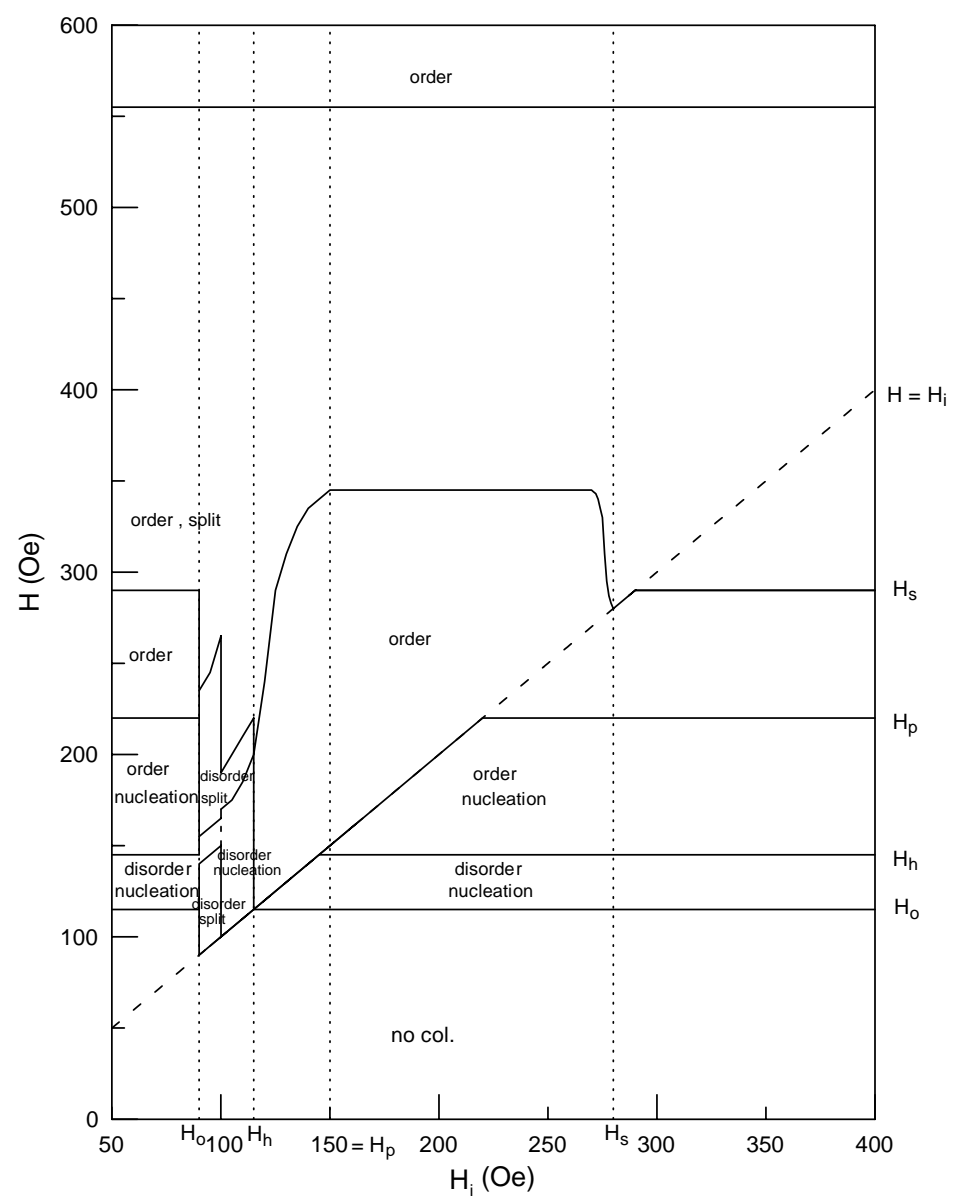

Fig. 1. Typical phase diagram of structural pattern formed inside a magnetic fluid film in $H-H_{\mathrm{i}}$ plane with $\mathrm{d} H / \mathrm{d} t=10 \mathrm{Oe} / \mathrm{s}$, $t_{\mathrm{d}}=3$ min. $H$ denotes the strength of the applied magnetic field and $H_{\mathrm{I}}$ represents the initial field strength. 
perpendicular magnetic fields, in the $H-H_{\mathrm{i}}$ plane, with duration time $t_{\mathrm{d}}=3 \mathrm{~min}$. Since $H_{\mathrm{i}}$ is less than $H_{0}$, the field strength required for the formation of magnetic columns, the magnetic fluid is monodispersed. The phenomena of the evolution of structural pattern are rich when the initial field strength is between $H_{0}$ and $H_{\mathrm{s}}$. When the initial field strength is between $H_{\mathrm{h}}$ and $H_{\mathrm{p}}$, where $H_{\mathrm{h}}$ is the field strength at which the ordered structure forms, and $H_{\mathrm{p}}$ corresponds to the field strength where the $H\left(H_{\mathrm{i}}\right)$ curve plateaus occur, $H$ becomes higher when $H_{\mathrm{i}}$ is higher. When the $H_{\mathrm{i}}$ is between $H_{\mathrm{p}}$ and $H_{\mathrm{s}}$, the curve in the $H-H_{\mathrm{i}}$ plane shows a plateau, where $H_{\mathrm{s}}$ is the field strength at which magnetic columns start to split.

Fig. 2 gives the enlarged partial processes of structural formation in magnetic fluid films. It contains only the portion of $H\left(H_{\mathrm{i}}\right)$ curves between $H_{\mathrm{i}}=H_{\mathrm{h}}$ and $H_{\mathrm{i}}=H_{\mathrm{s}}$ in the $H-H_{\mathrm{i}}$ phase diagram, with different duration time $t_{\mathrm{d}}$. It shows that the processes with longer duration times require a higher magnetic field strength for the initially ordered structural pattern to enter the transition state. To further investigate the causes of the deviation of field strength required for an ordered structure to enter the transition state, the images of structure patterns of both the initial state and transition state are converted to the images in k-space by using FFT. Fig. 3 gives the images of the initial structural patterns $\left(H=H_{\mathrm{i}}\right)$ and the structural pattern of transition state $\left(H=H_{\mathrm{s}}\right)$ for $t_{\mathrm{d}}=1,5$, and $180 \mathrm{~s}$. The insets in these images are the corresponding images of FFT, from which the orderness of the structural pattern can be compared. It is clearly shown from (a), (c), and (e) that the longer the duration time, the better the orderness of the initially structural pattern. Also, from (b), (d), and (f), the better the orderness of ordered structure, the higher the splitting magnetic field, $H_{\mathrm{s}}$.

During the formative process, the magnetic columns form and move inside the film due to Brownian motion, and finally reach the equilibrium. Since the formation is
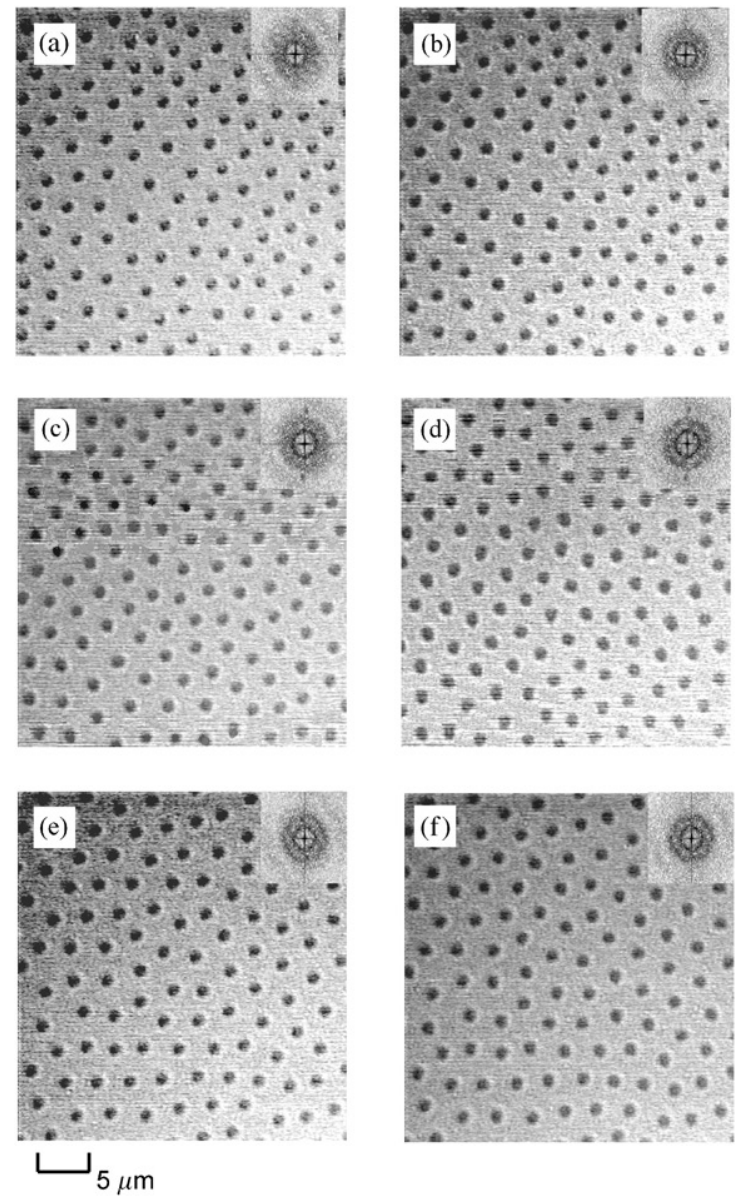

Fig. 3. Images of structural patterns in magnetic fluid thin films subjected to perpendicular magnetic field. The inset is the image of FFT transformation of the corresponding structural pattern, which is used to compare the orderness of the ordered structural pattern. (a), (b) $t_{\mathrm{d}}=1 \mathrm{~s}$ with $H_{\mathrm{i}}=190 \mathrm{Oe}$ and $H_{\mathrm{s}}=305$ Oe. (c), (d) $t_{\mathrm{d}}=5 \mathrm{~s}$ with $H_{\mathrm{i}}=190 \mathrm{Oe}$ and $H_{\mathrm{s}}=315 \mathrm{Oe}$. (e), (f) $t_{\mathrm{d}}=180 \mathrm{~s}$ with $H_{\mathrm{i}}=190 \mathrm{Oe}$ and $H_{\mathrm{s}}=345 \mathrm{Oe}$.

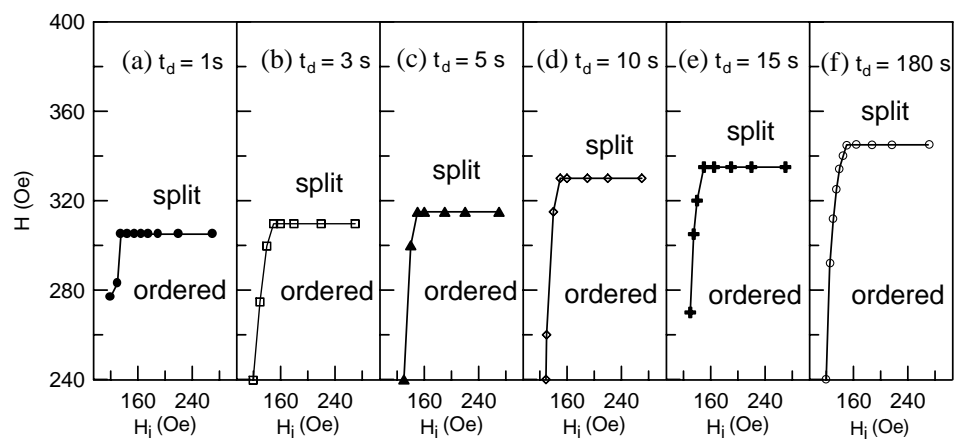

Fig. 2. Enlarged phase diagrams, containing only the portion between $H_{\mathrm{h}}$ and $H_{\mathrm{s}}$, of structural pattern formed inside a magnetic fluid film in $H-H_{\mathrm{i}}$ plane with $H_{\mathrm{i}}=190 \mathrm{Oe} / \mathrm{s}$ and $\mathrm{d} H / \mathrm{d} t=10 \mathrm{Oe} / \mathrm{s}$ for $t_{\mathrm{d}}$ 's of: (a) $1 \mathrm{~s}$; (b) $3 \mathrm{~s}$; (c) $5 \mathrm{~s}$; (d) $10 \mathrm{~s}$; (e) $15 \mathrm{~s}$; and (f) $180 \mathrm{~s}$. 
due to Brownian motion, the duration time in the formative process plays an important role in the status of the initially ordered structure. If the duration time were set to be long enough, the ordered structure would become nearly perfect. At this stage, each magnetic column is acted upon by the largest repulsive forces from its neighbors. Thus, it is difficult to change its state of an ordered structure into the transition state. Both Figs. 2 and 3 provide the evidence for this argument.

\section{Conclusion}

Various degrees of the initially ordered structure in magnetic fluid film under a perpendicular magnetic field can be created by setting different duration times $t_{\mathrm{d}}$ at a given initial field strength $H_{\mathrm{s}} \geqslant H_{\mathrm{i}} \geqslant H_{\mathrm{h}}$. The longer the duration time, the better the orderness of the structure. Furthermore, the field strength required for the ordered structure to enter the transition state becomes higher as the orderness of the initial structure becomes better.

\section{Acknowledgements}

This work was supported by the National Science Council of ROC under grant Nos. NSC89-2212-E-212021 and NSC89-2112-M-003-040.

\section{References}

[1] G.A. Jones, H. Niedoba, J. Magn. Magn. Mater. 73 (1988) 33.

[2] H. Wang, Y. Zhu, C. Boyd, W. Luo, A. Cebers, R.E. Rosensweig, Phys. Rev. Lett. 72 (1994) 1929.

[3] Chin-Yih Hong, I.J. Jang, H.E. Horng, C.J. Hsu, Y.D. Yao, H.C. Yang, J. Appl. Phys. 81 (1997) 4275.

[4] Herng-Er Horng, S.Y. Yang, S.L. Lee, Chin-Yih Hong, H.C. Yang, Appl. Phys. Lett. 79 (2001) 350.

[5] Chin-Yih Hong, Herng-Er Horng, F.C. Kuo, S.Y. Yang, H.C. Yang, J.M. Wu, Appl. Phys. Lett. 75 (1999) 2196.

[6] J. Cernak, P. Macko, J. Magn. Magn. Mater. 123 (1993) 107.

[7] Chin-Yih Hong, C.A. Chen, Chun-Hui Chen, H.E. Horng, S.Y. Yang, H.C. Yang, Appl. Phys. Lett. 79 (2001) 2360. 